\title{
From Molecular to Supramolecular: An Exploration into the Modes of Self- assembly in Conformationally Locked Polycyclitols
}

\author{
Goverdhan Mehta* and Saikat Sen
}

\begin{abstract}
This brief account highlights the notable findings of our investigation into the supramolecular chemistry of conformationally locked polycyclitols in the solid state. The study was aimed at analyzing the crystal packing and unraveling the modalities of non-covalent interactions (particularly, intramolecular vis-à-vis intermolecular $\mathrm{O}-\mathrm{H} \cdots \mathrm{O}$ hydrogen bonds) in polyols. The know-how obtained thereof, was successfully utilized to engineer selfassemblies of designer polycyclitols, having hydrogen bond donors and acceptors fettered onto a trans-decalin scaffold. The results seek to draw particular attention to the intrinsic attribute of this rigid carbocyclic framework to lock functional groups into spatially invariant positions and bring potential intramolecular hydrogen bonding partners into favorable interaction geometry to engender predictability in the self-assembly patterns.
\end{abstract}

Keywords: Conformational locking · Crystal engineering · Hydrogen bonding · Polycyclitols · Supramolecular chemistry

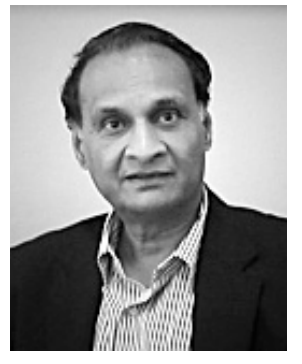

Goverdhan Mehta was born in Jodhpur, India. After his $\mathrm{PhD}$ from the University of Poona and postdoctoral research at the Michigan and the Ohio State Universities, he held faculty appointments at IIT Kanpur, University of Hyderabad (Vice Chancellor, 19941998) and the Indian Institute of Science, Bangalore (Director, 1998-2005). He is presently National Research Professor and Lilly-Jubilant Chair. He has published extensively, and is a recipient of numerous academic and civilian honours/awards and honorary doctorate degrees. He is a Fellow of the Royal Society (FRS) as well as of other Academies in India and abroad. His research interests encompass synthesis of bioactive natural products, creation of new and aesthetically pleasing molecular entities and incisive probing of stereo-electronic effects.

\section{Introduction}

Supramolecular chemistry, aptly termed by Lehn as the study of molecular sociology, is the chemistry of the intermolecular bond, focusing on the structures and functions of 'supermolecules' - chemical systems formed by the association between two or more molecular components. ${ }^{11]}$ While interrelated, supramolecular chemistry forges beyond the domain of traditional molecular chemistry and blends the comprehensive resources of molecular chemistry with a designed control of the intermolecular interactions. Not surprisingly, it has been stated that supermolecules are to molecules and the intermolecular bond what molecules are to atoms and the covalent bond..$^{[1 \mathrm{a}]}$ In the realm of molecular crystals, the focus of supramolecular chemistry converges with that of a rather recent, but rapidly emerging interfacial subdiscipline of crystal engineering. ${ }^{[2]}$ Coined nearly four decades ago in conjunction with photodimerization reactions in crystalline cinnamic acids, ${ }^{[3]}$ the term 'crystal engineering' has since then broadened its expanse considerably and is, at present, most appropriately defined as "the understanding of intermolecular interactions in the context of crystal packing and the utilization of such understanding in the design of new solids with desired physical and chemical properties".[2a]

A basic paradigm can be therefore expounded from any crystal engineering strategy, viz. design molecular building blocks with a prior knowledge of the pos- sible non-covalent interactions, such that they are pre-ordained to self-assemble in a manner that leads to the desired crystal structure. Like tools in a crystal engineer's kit, non-covalent interactions in molecular crystals are varied in nature, and broadly include the directional hydrogen bonds (and other dipole-dipole interactions, i.e. $\stackrel{\delta-}{\mathrm{D}}-\mathrm{X} \cdot \cdots \stackrel{\delta-}{\mathrm{A}}$, where $\mathrm{X} \neq \mathrm{H}$ ) and isotropic van der Waals forces. ${ }^{[2 a, 4,5]}$ Among the archetypes of hydrogen bonding interactions, the classical $\mathrm{O}-\mathrm{H}$... O hydrogen bond, well recognized to be vital for life itself, has been thoroughly studied and extensively documented. [6]

Many biologically important polyhydroxylated compounds, such as carbohydrates and inositols, have long served as model systems for the systematic study of $\mathrm{O}-\mathrm{H} \cdots \mathrm{O}$ hydrogen bonds.[6a,b,d] The large database of accurately determined crystal structures of these biomolecules has, over the years, stimulated research in identifying the commonalities in the patterns of their $\mathrm{O}-\mathrm{H} \cdots \mathrm{O}$ hydrogen bonding in the solid state. In his seminal review 'Crystallographic Studies of Carbohydrates',[6a] Jeffrey pointed out that hydrogen bonding in carbohydrates tends to follow certain rules that are based on two primary concepts: (a) maximize the total number of hydrogen bonds per molecule, using as many donor/acceptor oxygens as possible, and (b) maximize cooperativity by forming as many finite and infinite chains of hydrogen bonds as possible.

The first of the two concepts could be validated in crystal structures of not only 
carbohydrates, but also a variety of monoand polyhydroxylated (polyols) species as well. In fact, it underlines a vital aspect of molecular self-assembly, known after Robertson as the principle of maximum hydrogen bonding. ${ }^{[7]}$ Non-covalent interactions, being much weaker than covalent bonds, derive their strength (the ability to control and direct crystal packing) from their sheer numbers. Hence, a crystal packing would generally be able to adjust itself in a way that ensures the involvement of as many available hydrogen atoms, bonded to electronegative groups, as possible in hydrogen bonding.

The second concept propounded by Jeffrey, ${ }^{[6 a]}$ namely, the occurrence of cooperative $\mathrm{O}-\mathrm{H} \cdots \mathrm{O}$ hydrogen bonding chains, was however shown by Taylor and Macrae to be strongly dependent on not only the number of $\mathrm{OH}$ groups in a particular polyhydroxylated species, but also the steric environment (the degree of substitution) around each hydroxyl functionality. ${ }^{[8]}$ The pivotal role played by steric effects in the crystal packing of a polyol was again underlined in a contemporary CSD study on vic-diols by Brock, wherein it was observed that the extent of $\mathrm{O}-\mathrm{H} \cdots \mathrm{O}$ bond formation itself depends on the degree of substitution of the vic-diol, an $\mathrm{R}^{2}{ }_{2}(10)$ dimer being the preferred motif in fully hydrogen bonded crystal structures. ${ }^{[9]}$ The importance of molecular bulk or shape in dictating the choice of $\mathrm{H}$-bonded motifs adopted by alcohols was also advanced by Bishop et al. in a separate CSD analysis on the occurrence of ladder-like supramolecular architectures in certain diols. [10]

While these generalizations serve as useful guidelines for understanding the predilection of polyols for certain $\mathrm{O}-\mathrm{H} \cdots \mathrm{O}$ hydrogen bonding motifs over others, predicting a precise model for the mode of molecular association through intermolecular $\mathrm{O}-\mathrm{H}$... $\mathrm{O}$ H-bonds in any polyhydroxylated molecule, to paraphrase Jeffrey, ${ }^{[6 a]}$ is a difficult proposition. ${ }^{[11]}$ In this context, orientational flexibility of the $\mathrm{C}-\mathrm{OH}$ groups poses a particular inconvenience, since a variable $\mathrm{C}-\mathrm{OH}$... O torsion-angle parameter has to be associated with each hydrogen bond considered. Hence, proposing the hydrogen bonded architecture in conformationally flexible polyols, having little or no constraints on the internal degrees of freedom, becomes even more complicated because the final spatial disposition of both the hydrogen bond donors and acceptors - the hydroxy groups - in the crystal structure of such molecules is often largely determined by the crystal packing itself.

Against this background, we decided to examine the possibility of limiting the $\mathrm{O}-\mathrm{H}$... O hydrogen bonding patterns in polyols by locking their hydroxy groups into pre-destined conformations, which would be unaffected by molecular packing. As would be elaborated in the succeeding sections, the primary impetus for this undertaking came from an in-house know-how of the means to polyhydroxylate a rigid trans-decalin framework - a synthetic stratagem which transforms aromatic hydrocarbons into a class of exotic constructs called conformationally locked polycyclitols.

\section{Polycyclitols, Conformational Locking and the trans-Decalin Scaffold}

From a purely chronological standpoint, the basic design of conformationally locked polycyclitols, such as the annulated inositols 1 (Fig. 1), ${ }^{[12]}$ was amply inspired by the preceding syntheses of the polycyclitols $\mathbf{2}$ and $\mathbf{3}$, in which the hydroxy functionalities were embedded in a cis-hydrindane and cis-decalin framework respectively. ${ }^{[13]}$ Spurred by the intriguing inhibitory activity of two stereochemically defined analogs of $\mathbf{2}$ and $\mathbf{3}, v i z .4$ and $\mathbf{5}$, specifically towards yeast $\alpha$-glucosidase, [13b] it appeared inherently interesting to investigate the properties of polycyclitols which might be conceived by transcribing the dense hydroxy pattern, present in $\mathbf{2}$ and $\mathbf{3}$, on to the rigid carbocyclic framework of either a trans-hydrindane or a transdecalin. Unlike $\mathbf{2}$ and $\mathbf{3}$, polyhydroxylated trans-hydrindanes/decalins of prototype 1 would evidently exhibit a spatial locking of the hydroxy substituents and thus define a novel class of polyols, namely the 'conformationally locked polycyclitols'.
Annulated inositols, wherein conformational locking of the hydroxy groups stemmed from an archetypical 9,10-dihydroxy-trans-decalin (or hydrindane) core, formed the first representative category of such polyhydroxylated molecules. ${ }^{[12]}$ While retaining the natural configuration of the parent inositol, these inositol analogues were destined to be frozen in a high-energy 'unnatural' conformation of the cyclitol moiety. For example, while myo-inositol, the most prevalent natural inositol diastereomer, exists in the stable conformation 6 with five equatorial and one axial hydroxy groups (5e/1a), ${ }^{[14]}$ the synthetic annulated myo-inositol 7 was found to be locked in a five axial and one equatorial conformation (5a/1e) (Fig. 1). ${ }^{[12]}$

Such ground-state axial-rich conformations of hydroxy groups were subsequently observed in various intermediates and end products, obtained along synthetic routes devised by our group for annulated hexoses 8,[15] inosito-inositols $\mathbf{9}^{[16]}$ and conjoined inositols 10 (Fig 2). [17] In each of these synthetic endeavors, the 9,10-dihydroxy-trans-decalin framework could be reliably employed as a prototypical path for locking the hydroxy substituents in spatial orientations which might not have been realized otherwise.

From annulated to conjoined inositols, our endeavors to evolve a general synthetic protocol for the stereo- and regioselective polyhydroxylation of the trans-decalin framework stemmed from the expectation that such a tactic would lead to novel variants of naturally occurring cyclitols with hydroxy groups destined to be locked in an unnatural axial-rich conformation. It was conjectured that such a conformational

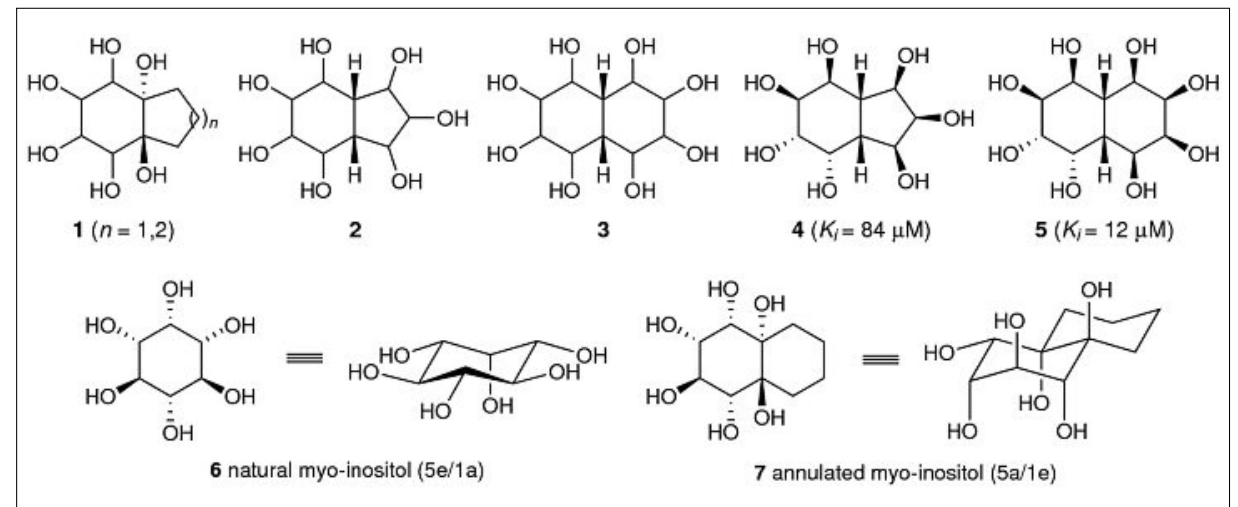

Fig. 1.

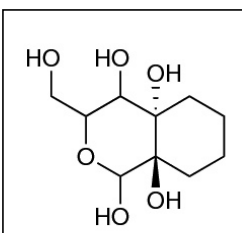

8

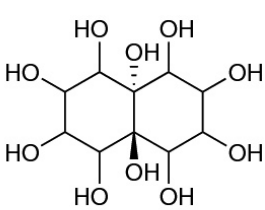

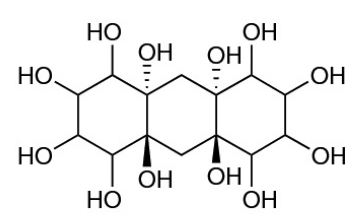

10

Fig. 2. 


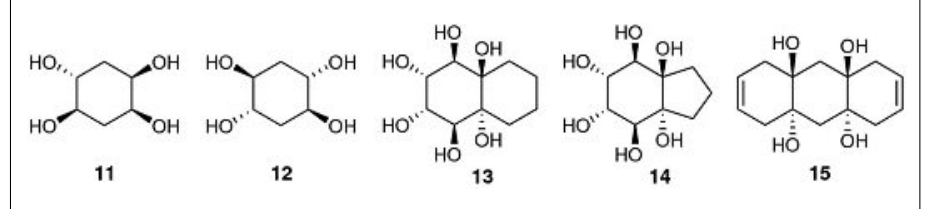

Fig. 3.

locking of hydroxy groups would lead to a significant alteration in the reactivity pattern and molecular recognition profile of the cyclitol moiety. Much in the way of a corollary, it became evident that conformationally locked polycyclitols would also exhibit $\mathrm{O}-\mathrm{H} \cdots \mathrm{O}$ hydrogen bonding characteristics and self-assembling patterns in the solid state, distinctly different from those observed for their natural congeners. It was our desire to understand the manner in which such spatial locking of the hydroxy groups into axial-rich conformations would express itself in the molecular packing that goaded us to investigate the solid-state supramolecular chemistry of conformationally locked polycyclictols.

\section{Spatial Locking of Hydroxy Groups by Design and its Implication in Engineering Self- assemblies of Polycyclitols}

It comes as a natural inference that an axial-rich disposition of the hydroxy groups in a polyhydroxylated molecule should automatically bring the 1,3-syn $\mathrm{OH}$ functionalities into a geometry favorable for the formation of intramolecular $\mathrm{O}-\mathrm{H}$... O hydrogen bonds. However, it is also well-known that a ground-state syndiaxial conformation of hydroxy functionalities is energetically unfavorable for most of the naturally occurring polyols, so that intermolecular $\mathrm{O}-\mathrm{H} \cdots \mathrm{O}$ hydrogen bonds, either to neighboring molecules or solvent, are preferred over the intramolecular ones and observed ubiquitously in the crystal structures of such molecules. [6]

This point was re-iterated in our study of solid-state self-assemblies in two diastereomeric cyclohexane-1,2,4,5-tetrols (11 and 12, Fig. 3). ${ }^{[18]}$ It is worth mentioning at this point that intramolecular $\mathrm{O}-\mathrm{H} \cdots \mathrm{O}$ hydrogen bonding has not been observed even in the crystal structure of epi-inosi-

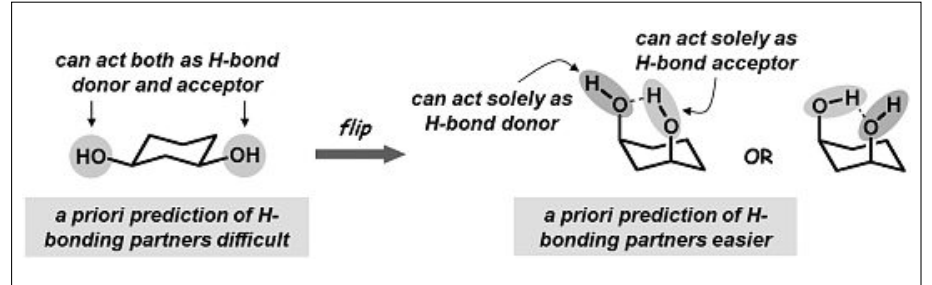

Fig. 4. Formation of intramolecular $\mathrm{O}-\mathrm{H} \cdots \mathrm{O}$ hydrogen bonds between 1,3-syndiaxial hydroxy groups can preordain the positions of intermolecular $\mathrm{O}-\mathrm{H} \cdots \mathrm{O}$ hydrogen bond donors and acceptors in a polyol molecule. This premise has been illustrated here with the two possible conformations of 1,3-cyclohexanediol.

tol where a 1,3-syndiaxial conformation of hydroxy groups is unavoidable. ${ }^{[6 a, 19]}$ In comparison, our studies on the crystal structures of the annulated inositols $(\mathbf{7}, 13$ and 14) ${ }^{[20]}$ and the unsaturated tetrol $\mathbf{1 5}^{[21]}$ revealed that the formation of intramolecular O-H...O hydrogen bonds between the 1,3-syndiaxial $\mathrm{OH}$ groups is an invariant feature of molecular packing in conformationally locked polycyclitols.

This implied in turn that reckoning the possible modes of crystal packing in a conformationally locked polycyclitol should presumably be less involved than other polyols because two hydroxy functionalities, participating in intramolecular H-bonding, would be preordained to function as either a donor or an acceptor (but not both) of intermolecular O-H...O hydrogen bonds in the crystal structure (Fig. 4).

But can locking of hydroxy groups into axial-rich conformations and rendering their spatial positions invariant to crystal packing truly lend predictability to the modes of $\mathrm{O}-\mathrm{H}$... O hydrogen bonding in polycyclitols? We sought to test the waters in the solid-state self-assemblies of three polyols 16-18 (Fig. 5). Synthesis of the polycyclitols 16-18 was carried out from readily available aromatic precursors (tetralin, naphthalene and anthracene respectively) via sequential epoxidation and stereoselective acid catalyzed ring opening on their Birch reduction products (see Scheme 1 for a representative example). ${ }^{[22]}$ The polycyclitols 16-18 were conceptualized with a common design element in mind, namely that all the hydroxy groups in them would be destined to participate in intramolecular $\mathrm{O}-\mathrm{H} \cdots \mathrm{O}$ hydrogen bonding.

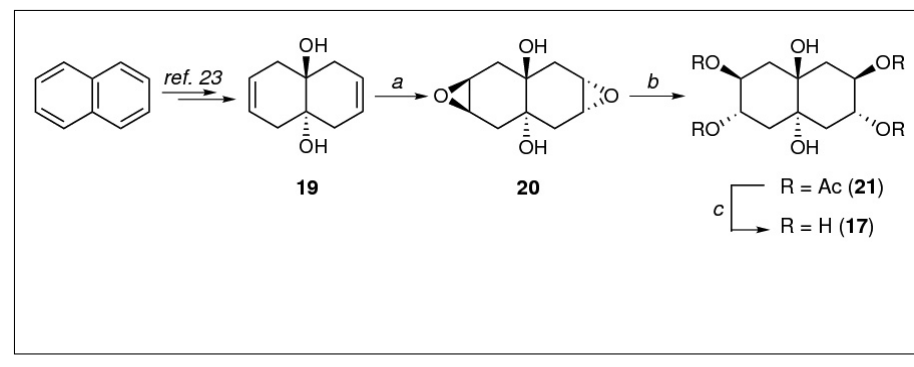

Scheme 1. Reagents and Conditions: ${ }^{[22]}$ (a) $m$ CPBA, $\mathrm{CH}_{2} \mathrm{Cl}_{2}, 0^{\circ} \mathrm{C}$ $\rightarrow \mathrm{RT}, 3 \mathrm{~h}, 87 \%$; (b) (i) pTSA, moist DCM, RT; (ii) $\mathrm{Ac}_{2} \mathrm{O}$, pyridine, RT, 20 h $(65 \%$ over two steps); (c) $\mathrm{NaOMe}, \mathrm{MeOH}, 0^{\circ} \mathrm{C}$, $16 \mathrm{~h}$, quant.
Much like matching congenial partners or a game with LEGO $^{\circledR}$ bricks, the packing patterns in the crystal structures of 16-18 can be then conceptualized (in the simplest of the scenarios) essentially from the manner in which one of the intramolecularly H-bonded molecular motifs 22-28 (Fig. 6) chooses to be linked to its nearest neighbors through the four possible intermolecular $\mathrm{O}-\mathrm{H} \cdots \mathrm{O}$ hydrogen bonds. This observation not only simplified a qualitative visualization of the various packing patterns in 16-18, but also allowed us to propose, primarily based on steric considerations and crystal packing preferences of polyols from previously reported CSD studies (vide supra), ${ }^{66,8-10]}$ the packing motifs most likely to converge with the experimental results. Despite its qualitative nature, the $\mathrm{O}-\mathrm{H} \cdots \mathrm{O}$ H-bonding patterns, proposed for 16-18, were found to conform well with those observed experimentally for the tetrols $\mathbf{1 6}$ and $\mathbf{1 8}$, and even for the two polymorphic modifications of the hexol 17 (Fig. 7). [22-24]

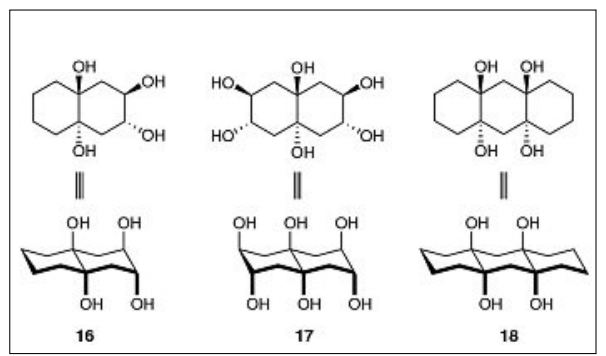

Fig. 5.

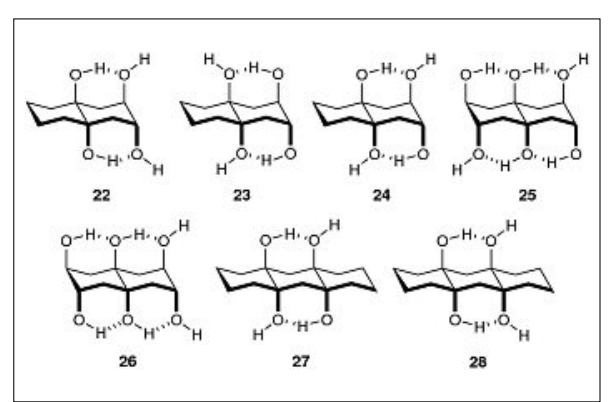

Fig. 6. The conformationally locked, intramolecularly $\mathrm{O}-\mathrm{H} \cdots \mathrm{O}$ hydrogen bonded molecular motifs. 


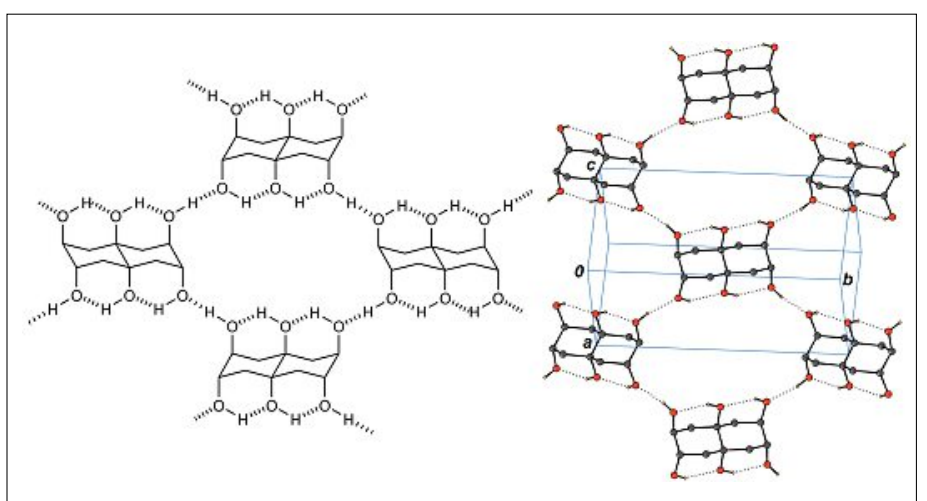

Fig. 7. (left) One of the most probable packing modes proposed for the hexol 17 by employing the centrosymmetric motif 25. ${ }^{[22]}$ That any molecular packing, generated from 25 rather than $\mathbf{2 6}$, would be more likely to be observed in the crystal structure of $\mathbf{1 7}$ was an obvious corollary of the conclusions drawn by Brock and Dunitz. A molecule, like 17, that possesses an inversion symmetry would prefer to crystallize in a centrosymmetric space group ${ }^{[25]}$ and occupy a crystallographic inversion center in such a case. ${ }^{[26]}$ By involving all donor/acceptor oxygens and incorporating infinite chains of $\mathrm{O}-\mathrm{H} \cdots \mathrm{O}$ bonds, the proposed $\mathrm{H}$-bonding pattern also follows the general trend of hydrogen bonding, observed in carbohydrates by Jeffrey. ${ }^{[6 a]}$ (right) Molecular packing observed experimentally in one of the polymorphs of $17^{[27]}$ (CrystEngComm. 2007, 9, 144 - Reproduced by permission of The Royal Society of Chemistry. Taken from ref. [24].

\section{Recognizing the Built-in Chemodifferentiation of Hydroxy Groups as means of Disabling the Peripheral O-H...O Hydrogen Bond Donors}

In the foregoing study, it is apparent that the simplistic nature of supramolecular assembly in $\mathbf{1 7}$ is characterized and effected by the end-to-end co-operative intramolecular $\mathrm{O}-\mathrm{H} \cdots \mathrm{O}-\mathrm{H}$ hydrogen bonding chain on both faces of the molecule. This observation led us to examine, much in the way of intellectual curiosity at the outset, the consequences of disabling the peripheral $\mathrm{O}-\mathrm{H} \cdots \mathrm{O}$ H-bond donors, in the form of the secondary hydroxy groups, in the hexol 17. To this end, the built-in chemodifferentiation between the hydroxy groups in $\mathbf{1 7}$ was exploited to protect the secondary hydroxy moieties selectively as their acyl derivatives. The acetyl and benzoyl protecting groups were selected for this purpose on grounds of the ease in their introduction (even in a sterically encumbered position), purification and the welldocumented crystallizability of the esters thus obtained. Inherent in this substrate design was the expectation that the presence of the two tertiary hydroxy groups as the sole $\mathrm{O}-\mathrm{H}$... O hydrogen bond donors in the hexol 17 would trigger the supramolecular assembly of the two tetra-acyl derivatives of $\mathbf{1 7}$, namely the tetra-acetate $\mathbf{2 1}$ and the tetrabenzoate $\mathbf{2 9}$, to evolve along two mutually exclusive pathways (Fig. 8).

The pathway 1 follows the hierarchy of the strength of the non-covalent interac-

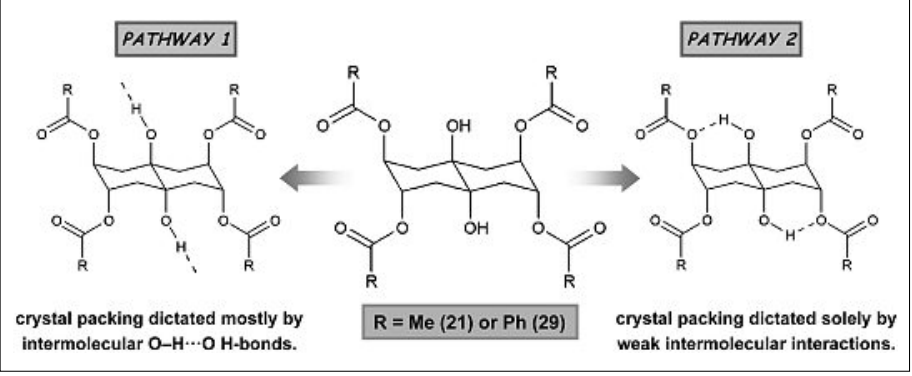

Fig. 8. The two principal and mutually exclusive modes of molecular packing in the tetra-acyl derivatives of the hexol 17.

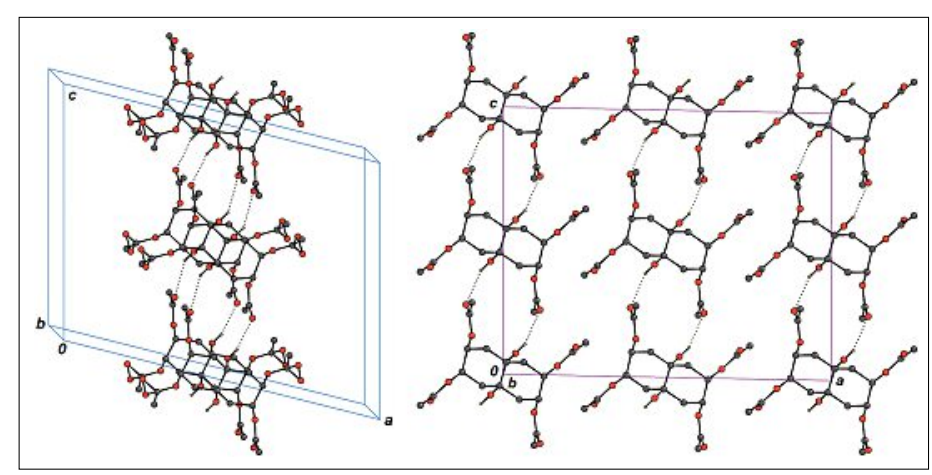

Fig. 9. Molecular packing in the $\alpha$ (left) and $\beta$ (right) forms of the tetraacetate 21. Note that in both polymorphs, the crystal packing is dictated by intermolecular $\mathrm{O}-\mathrm{H} \cdots \mathrm{O}$ hydrogen bonds, which link the molecules into tapes along the $c$ axis (Chem. Commun. 2009, 5981 - Reproduced by permission of The Royal Society of Chemistry. Taken from ref. [28].

tions available in $\mathbf{2 1}$ or $\mathbf{2 9}$, and opts for a crystal packing dictated mostly by intermolecular $\mathrm{O}-\mathrm{H}$-..O H-bonds, employing the lesser accessible tertiary hydroxy groups (Fig. 8). The pathway 2, on the other hand, relegates the central $\mathrm{OH}$ moieties to function as intramolecular $\mathrm{O}-\mathrm{H} \cdots \mathrm{O}$ H-bond donors to ester oxygens and settles for a self-assembly dictated solely by weaker intermolecular interactions, involving the alkyl/aryl groups of the ester moieties (Fig. 8 ). It is to be noted that while proposing the two modes of $\mathrm{O}-\mathrm{H} \cdots \mathrm{O}$ hydrogen bonding, it was assumed that either of the two $\mathrm{C}_{2 h}$ symmetric tetra-acyl derivatives (21 and 29) will occupy a crystallographic inversion center in its experimentally observed crystal structure.[26]

Irrespective of the crystallization conditions employed, a pure sample of the tetraacetate $\mathbf{2 1}$ was found to crystallize exclusively along pathway 1 , albeit in two enantiotropic polymorphic modifications, ${ }^{[27]}$ one obtainable at room temperature $\left(\alpha\right.$ form) and the other at $-20{ }^{\circ} \mathrm{C}(\beta$ form) (Fig. 9). ${ }^{28]}$ Behaving much like a temperature-guided molecular switch, the tetraacetate $\mathbf{2 1}$ could shift reversibly between the $\alpha$ and $\beta$ forms in response to changes in the ambient temperature. Thus, the $\alpha$ form converted at $-4{ }^{\circ} \mathrm{C}$ to the denser $\beta$ form, which displayed an unusual kinetic stability till $67^{\circ} \mathrm{C}$ and transformed back to the $\alpha$ form beyond this temperature.
It was however possible to goad $\mathbf{2 1}$ into crystallizing along the pathway 2 through preferential inhibition of pathway 1 in presence of a 'tailor-made' additive ${ }^{[29]}$ namely, a diastereomeric tetraacetate $\mathbf{3 0}$, obtained serendipitously en route to $\mathbf{2 1}$ via an apparent breakdown of the FürstPlattner rule (Scheme 2). ${ }^{[30,31]}$ As a matter of fact, our rationale behind employing 30 as a nucleation inhibitor to access the elusive third polymorphic modification of 21 (the $\gamma$ form) had more to do with the observed uncanny similarity between the crystal packing of $\mathbf{3 0}$ and the two dimorphs of 21, rather than the diastereomeric relationship between 21 and 30 (Fig. 10, left). Crystal structure of the $\gamma$ form confirmed our expectations - with the hydroxy groups engaged in intramolecular $\mathrm{O}-\mathrm{H}$... O hydrogen bonding, the tetraacetate molecules in the $\gamma$ form were held in place solely via the weaker C-H...O hydrogen bonds (Fig. 10, right). [31]

With increased sequestering of the central hydroxy groups by the peripheral benzoate moieties in the tetrabenzoate $\mathbf{2 9}$, crystal packing in $\mathbf{2 9}$ preferred to follow exclusively pathway 2 as expected. Similar to the $\gamma$ form of the tetraacetate $\mathbf{2 1}$, the hydroxy groups participated in intramolecular $\mathrm{O}-\mathrm{H} \cdots \mathrm{O}$ hydrogen bonding, while intermolecular $\mathrm{C}-\mathrm{H}$... O hydrogen bonds linked the molecules in the crystal structure of 29 (Fig. 11). [32] 


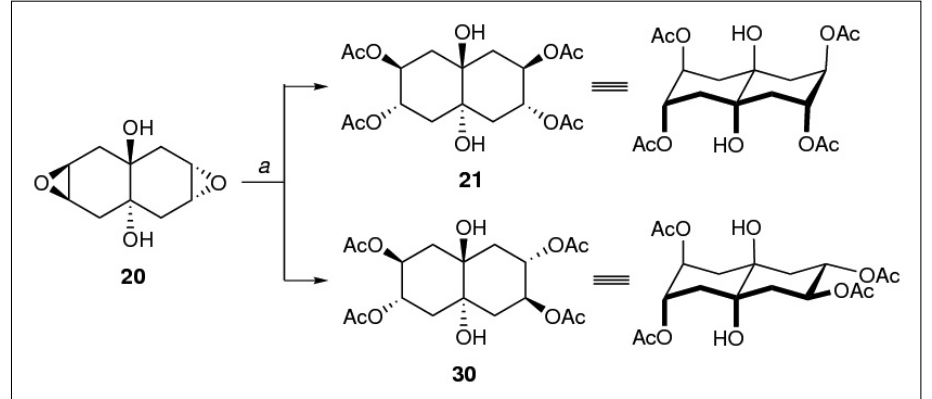

Scheme 2. Reagents and Conditions: ${ }^{[31]}$ (a) (i) $10 \% \mathrm{v} / \mathrm{v} \mathrm{AcOH}$ (aq.), RT, 48 $\mathrm{h}$; (ii) $\mathrm{Ac}_{2} \mathrm{O}, \mathrm{DMAP}, \mathrm{RT}, 10 \mathrm{~h}$ [21 : $\mathbf{3 0}=9$ : 1; yields over two steps: $44 \%$ (21) and $5 \%(30)]$.

\section{Extending the Concept of Conformational Locking to the Study of Non-covalent Interactions Involving 'Organic Fluorine'}

Having successfully exploited the trans-decalin scaffold to predictably bring hydroxy groups into a geometry congenial for intramolecular $\mathrm{O}-\mathrm{H} \cdots \mathrm{O}$ hydrogen bonding, it came as a natural extrapolation that the concept of conformation locking can be employed as a simple, but effective, maneuver to enforce propinquity (a 1,3-syndiaxial relationship) between a fluro and a hydroxy group, and thus evaluate unambiguously the capability of 'organic fluorine' to serve as an acceptor for $\mathrm{O}-\mathrm{H} \cdots \mathrm{F}$ hydrogen bonding. This proposition had a particular contemporary relevance on account of the wide ranging practical applications of organofluorine compounds ${ }^{[33]}$ and the on-going controversy that resides over the ability of organic fluorine to function as a hydrogen bond acceptor. ${ }^{[34]} \mathrm{We}$ believed that a minor tweak in the synthetic stratagem, leading to $\mathbf{1 7}$ and $\mathbf{1 8},,^{[22]}$ should allow introduction of a fluorohydrin moiety in the trans-decalin framework and afford access to a novel class of molecular probes, capable of bringing clarity to the role of fluorine in a self-assembly.

Accordingly, three fluorinated polycyclitols 31-33 (Fig. 12) were crafted with the specific intent of investigating the capability of covalently bonded fluorine to engage itself in $\mathrm{C}\left(s p^{3}\right)-\mathrm{F} \cdots \mathrm{H}-\mathrm{X}\left(s p^{3}\right)$ (X $=\mathrm{O}$ and/or $\mathrm{C}$ ) $\mathrm{H}$-bonding, in presence of its isostere, the hydroxyl group. ${ }^{[35,36]}$ Conformationally locked with well-

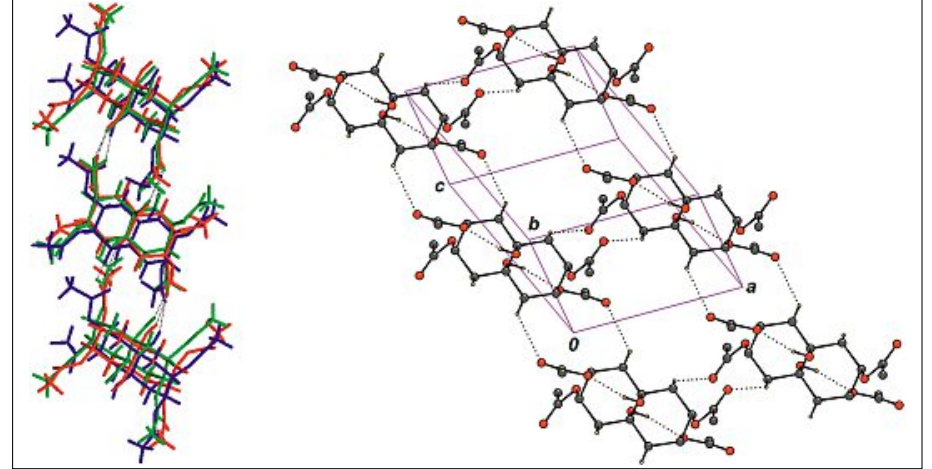

Fig. 10. (left) Molecular overlay diagram of packing in $\mathbf{3 0}$ (blue) and the $\alpha$ (red) and $\beta$ (green) forms of 21. (right) Crystal packing in the $\gamma$ form of 21 [Reprinted from Tetrahedron 2009, 65, 9713 with permission from Elsevier $]^{[31]}$ defined spatial disposition of functional groups, all the three fluorinated polycyclitols 31-33 bore a fluorohydrin moiety, embedded in a rigid trans-decalin framework. In 31 and 33, it was conceived that the presence of a hydroxyl donor in a favorable 1,3-syndiaxial relationship to a fluoro group on one side and a hydroxyl group on the other would allow an unambiguous comparison between the two isosteric functionalities $(\mathrm{C}-\mathrm{OH}$ and $\mathrm{C}-\mathrm{F})$ to serve as acceptors for intramolecular hydrogen bonds $(\mathrm{O}-\mathrm{H} \cdots \mathrm{O}$ and purported $\mathrm{O}-\mathrm{H} \cdots \mathrm{F}$ respectively). The difluorodiol $\mathbf{3 2}$ was sought to serve as a control to assess the change in the $\mathrm{C}-\mathrm{F} \cdots \mathrm{H}-\mathrm{X}$ interactions (if any) which might be observed upon incorporating the peripheral secondary hydroxyl groups in $\mathbf{3 3}$.

As indicated above, the synthetic strategy leading to the three fluorinated polycyclitols 31-33 was chalked out quite in

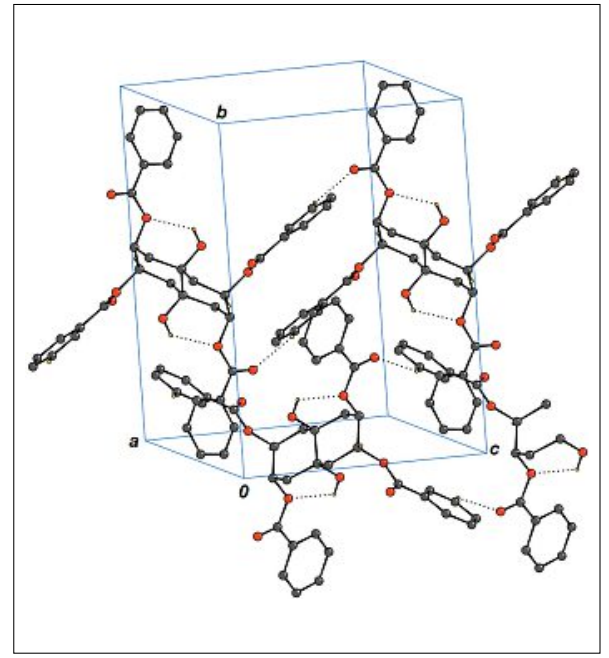

Fig. 11. Molecular packing in the tetrabenzoate 29 (Acta Crystallogr., Sect. C: Cryst. Struct. Commun. 2010, C66, o59 - Reproduced by permission of the International Union of Crystallography. ${ }^{[32]}$

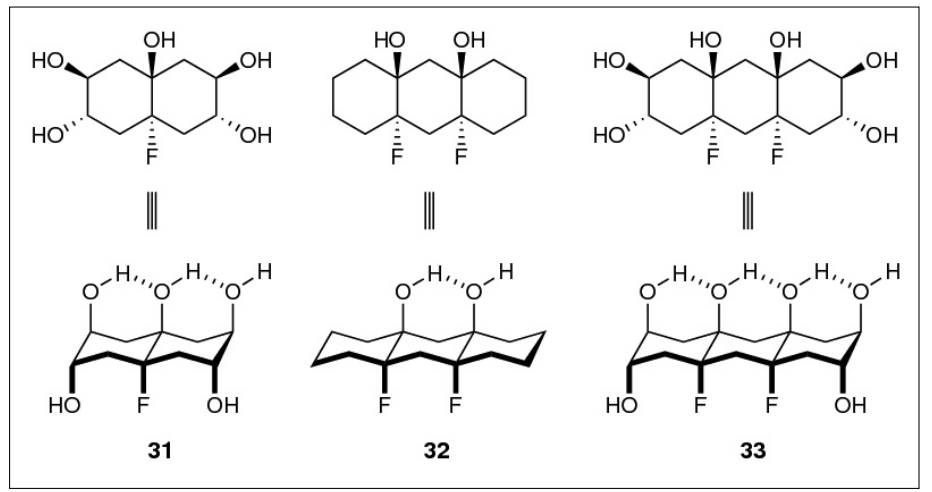

Fig. 12.

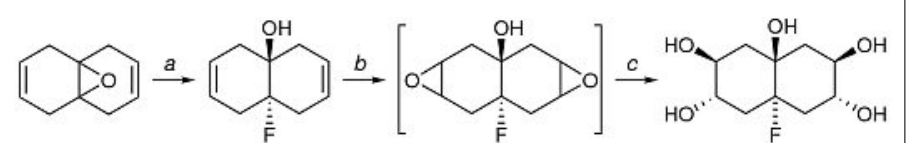

34

35

31

Scheme 3. Reagents and Conditions: ${ }^{[36]}$ (a) pyridine poly(hydrogen fluoride), THF, RT, 12 h, 97\%; (b) $m$ CPBA (2.1 equiv.), $\mathrm{CH}_{2} \mathrm{Cl}_{2}, \mathrm{RT}, 1 \mathrm{~h}, 78 \%$ (overall); (c) $10 \% \mathrm{AcOH}$ (aq.), THF, $50-60{ }^{\circ} \mathrm{C}, 3$ days, $75 \%$ after re-crystallization from 1:3 methanol-ethyl acetate.

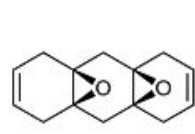

36

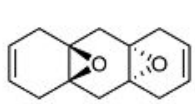

37
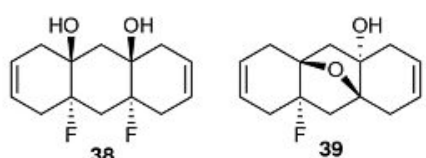

Fig. 13. 


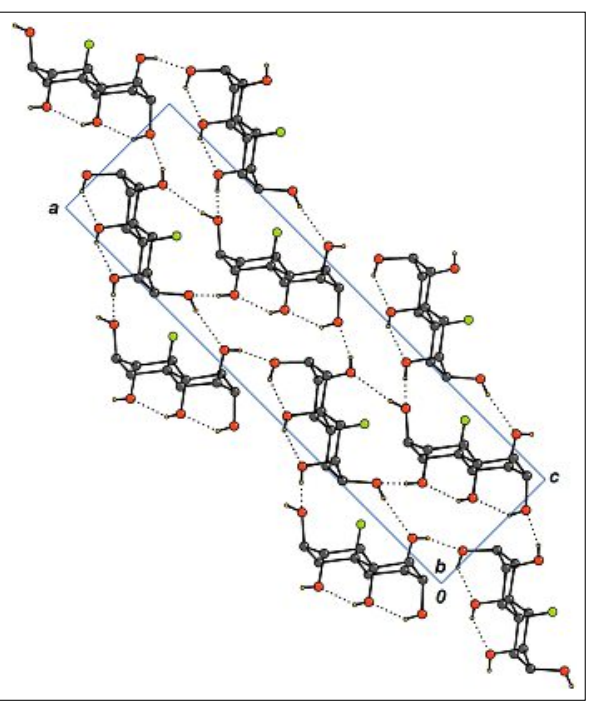

Fig. 14. Molecular packing in the monofluoropentol 31. The dotted lines indicate the $\mathrm{O}-\mathrm{H} \cdots \mathrm{O}$ hydrogen bonds [Reprinted from Eur. J. Org. Chem. 2010, 3387-3394 with permission from John Wiley and Sons]. ${ }^{[36]}$

analogy to that followed for the preparation of the polycyclitols 17 and 18. ${ }^{[22]}$ As a representative example, synthesis of the monofluoropentol 31 commenced with pyridine-poly(hydrogen fluoride) mediated ring opening of the epoxide $34,{ }^{[37]} \mathrm{ob}-$ tained from isotetralin via regioselective electrophilic epoxidation. ${ }^{23 \mathrm{c}]}$ Treatment of the resulting trans-fluorohydrin $\mathbf{3 5}$ with $m$ CPBA, followed by mild acid mediated ring opening in the diastereomeric mixture of diepoxides (36) thus obtained, ${ }^{[38]}$ furnished the desired monofluoropentol $\mathbf{3 1}$ as the sole product (Scheme 3). ${ }^{[39]}$ The stereochemical convergence, occurring during the formation of $\mathbf{3 1}$ from $\mathbf{3 6}$, conformed to our earlier observations in the synthesis of $\mathbf{1 6}-\mathbf{1 8},{ }^{[22]}$ and could be anticipated on the basis of the conformational rigidity of the trans-decalin framework and the FürstPlattner rule. [30]

A synthetic route, similar to the one engendering $\mathbf{3 1}$ from the epoxide $\mathbf{3 4}$, led us to obtain the difluorodiol 32 and the difluorohexol 33 from the syn-diepoxide 36 (conveniently prepared, along with its anti-diastereomer 37, from 1,4,5,8,9,10-hexahydroanthracene ${ }^{[22,40]}$ ) via the common intermediate 38. ${ }^{[36,41]}$ Unlike the syn-diepoxide 36, which underwent bis-fluorination in pyridine poly(hydrogen fluoride) with complete regio- and streocontrol to furnish the unsaturated difluorohexol $\mathbf{3 8}$ as the sole product, ${ }^{[36]}$ its sibling $\mathbf{3 7}$ afforded the tetracyclic monofluoroalcohol 39 (Fig. 13) as the major component of the product mixture via a tandem HF-mediated epoxide ring opening and transannular oxacyclization. ${ }^{[42]}$

While the presence of intramolecular $\mathrm{O}-\mathrm{H} \cdots \mathrm{O}$ hydrogen bonds between the 1,3-syndiaxial hydroxy groups was ex-

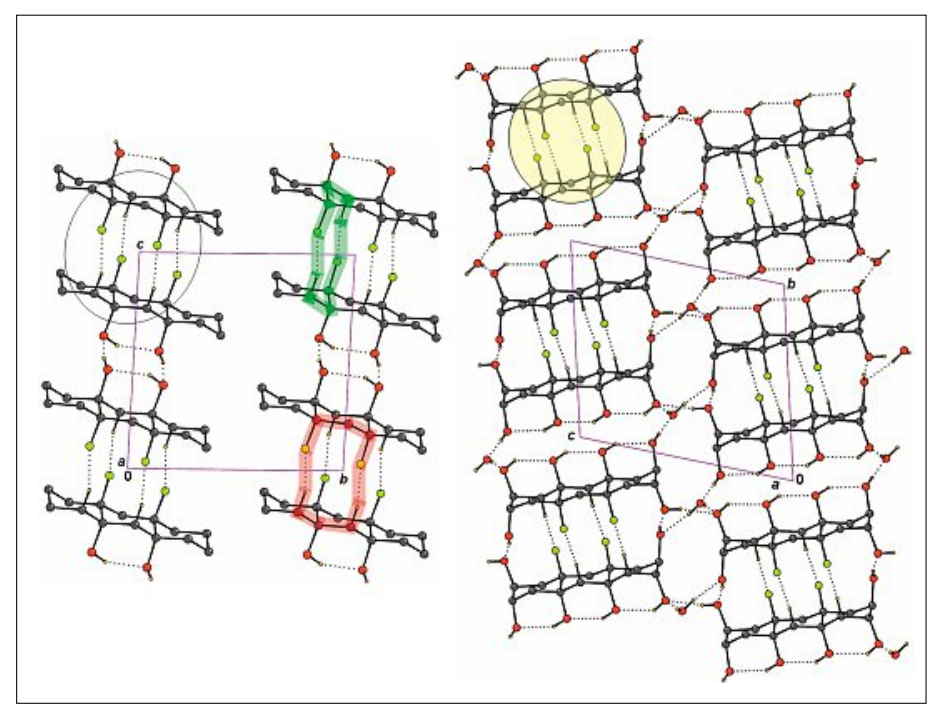

Fig. 15. (left)

Molecular packing in the difluorodiol $\mathbf{3 2}$, showing how four intermolecular $\mathrm{C}-\mathrm{H} \cdots \mathrm{F}$ hydrogen bonds forms a part of a $\mathrm{R}_{2}^{2}(10) \mathrm{H}$-bonding motif (encircled). This centrosymmetric supramolecular recognition unit is observed even in the molecular packing in the difluorohexol $\mathbf{3 3}$ (right) [Reprinted from Eur. J. Org. Chem. 2010, 3387-3394 with permission from John Wiley and Sons]. ${ }^{[36]}$

pectedly an easily discernible feature of molecular packing in 31-33, no $\mathrm{O}-\mathrm{H} \cdots \mathrm{F}$ interactions, either intra- or inter, could be gleaned in the crystal structures of 31-33. As expected from any polyhydroxylated molecule, the solid-state self-assembly of all the three fluorinated polycyclitols 31-33 strove to maximize the $\mathrm{O}-\mathrm{H} \cdots \mathrm{O}$ $\mathrm{H}$-bonds possible. Indeed, the extensive $\mathrm{O}-\mathrm{H} \cdots \mathrm{O}$ hydrogen bonding network in 31-33 involved all the donor oxygen atoms and was the sole interaction controlling the molecular packing in the monofluoropentol 31, wherein short $\mathrm{H} \cdots \mathrm{F}$ contacts resulted merely from the compressive forces generated by the intricate $\mathrm{O}-\mathrm{H} \cdots \mathrm{O}$ H-bonding network (Fig. 14).

However, the crystal structures of the difluorinated polyols $\mathbf{3 2}$ and $\mathbf{3 3}$ presented an interesting adjustment to fulfill the principle of maximum hydrogen bonding. ${ }^{[7]}$ Despite the presence of stronger $\mathrm{O}-\mathrm{H} \cdots \mathrm{O}$ $\mathrm{H}$-bonds, $\mathrm{C}-\mathrm{H} \cdots \mathrm{F}$ hydrogen bonds were observed - not just in effect, but as the constituent interactions of a conserved threedimensional centrosymmetric supramolecular recognition unit as well (Fig. 15). It is pertinent to mention at this point that the clustering of fluoroalkyl substituents, as noted in the crystal structures of $\mathbf{3 2}$ and 33, has been observed even in aggregates of fluorinated peptides and other fluorine based materials, and has been suggested to lend increased stability to the supramolecular assembly. ${ }^{[43]}$ In the self-assemblies of the difluorinated polycyclitols $\mathbf{3 2}$ and 33, this enhanced stability might be contributed by the antiparallel arrangement of the $\mathrm{C}-\mathrm{F}$ dipoles in the common centrosymmetric $\mathrm{C}-\mathrm{H} \cdots \mathrm{F}$ recognition unit.

\section{Summary and Outlook}

In hindsight, we have successfully demonstrated that a trans-decalin derived scaffold can be employed as a versatile plat- form to design and craft diverse molecular entities with desired functional attributes. For the study of non-covalent interactions, one may utilize this rigid carbocyclic scaffold not just to lock functional groups into spatial positions that will remain unaffected by crystal packing, but bring, as 'matchmakers', potential hydrogen bonding partners into a favorable intramolecular interaction geometry as well. In the case of polycyclitols, this tactic - namely, maximize the possible intramolecular $\mathrm{O}-\mathrm{H} \cdots \mathrm{O}$ hydrogen bonds in order to minimize the number of intermolecular O-H...O hydrogen bonds needed to be considered - can viewed upon as a viable modus operandi for 'scaffold based crystal engineering'. It is equally feasible to exploit the in-built chemodifferentiation between the central and peripheral functional groups on a trans-decalin framework to sequester or completely disable an activated hydrogenbond donor, and allow the crystal packing to be dictated by weaker interactions alone. Indeed, there is much room for extending our findings towards the study of hydrogen bonding and self-assembling preferences in molecules having not only hydroxyl, but also other functional groups (such as $\mathrm{NH}_{2}$, $\mathrm{COOH}$ and halogens) as well.

\section{Acknowledgement}

The present account is largely based on the Ph.D. Thesis (2010) of Dr. Saikat Sen and the experimental work was carried out at the Indian Institute of Science, Bangalore. GM thanks Government of India for the award of the National Research Professorship and acknowledges the current research support from Eli Lilly and Jubilant-Bhartia Foundations.

Received: October 7, 2012

[1] (a) J.-M. Lehn, Angew. Chem. Int. Ed. Engl. 1988, 27, 89; (b) J.-M. Lehn, Angew. Chem. Int. Ed. Engl. 1990, 29, 1304; (c) J.-M. Lehn, Science 1993, 260, 1762; (d) J.-M. Lehn, in 'Supramolecular Chemistry: Concepts and Perspectives: a personal account', Wiley- 
VCH: Weinheim, 1995; (e) 'Comprehensive Supramolecular Chemistry', Vols. 1-10, Eds. J. L. Atwood, J. E. D. Davies, D. D. MacNicol, F. Vögtle, J.-M. Lehn, Pergamon, Oxford, 1996; (f) Special issue on molecular recognition, Chem. Rev. 1997, 97, 1231; (g) J. W. Steed, J. L. Atwood, in 'Supramolecular Chemistry', Wiley, Chichester, 2000; (h) Special issue on supramolecular chemistry and self-assembly, Proc. Natl. Acad. Sci. U.S.A. 2002, 99, 4755.

[2] (a) G. R. Desiraju, in 'Crystal Engineering. The Design of Organic Solids', Elsevier: Amsterdam, 1989; (b) G. R. Desiraju, Angew. Chem. Int. Ed. Engl. 1995, 34, 2311; (c) G. R. Desiraju, Chem. Commun. 1997, 1475; (d) C. B. Aakeröy, Acta Crystallogr., Sect. B: Struct. Sci. 1997, B53, 569; (e) D. Braga, F. Grepioni, G. R. Desiraju, Chem. Rev. 1998, 98, 1375; (f) A. Nangia, G. R. Desiraju, Acta Crystallogr., Sect. A: Found. Crystallogr. 1998, A54, 934; (g) 'Crystal Engineering. From Molecules and Crystals to Materials', Eds. D. Braga, F. Grepioni, F. A. G. Orpen, Kluwer, Dordrecht, 1999; (h) M. J. Zaworotko, in 'Crystal Engineering: The Design and Application of Functional Solids', Eds. K. R. Seddon, M. J. Zaworotko, NATO, ASI series, Kluwer, Dordrecht, 1999; (i) B. Moulton, M. J. Zaworotko, Chem. Rev. 2001, 101, 1629; (j) M. D. Hollingsworth, Science 2002, 295, 2410; (k) D. Braga, Chem. Commun. 2003, 2751; (1) D. Braga, L. Brammer, N. R. Champness, CrystEngComm 2005, 7, 1; (m) G. R. Desiraju, Angew. Chem. Int. Ed. 2007, 46, 8342.

[3] (a) G. M. J. Schmidt, Pure Appl. Chem. 1971, 647; (b) 'Solid State Photochemistry. A Collection of Papers by G. M. J. Schmidt and his Collaborators', Monographs in Modern Chemistry, Vol. 8, Ed. D. Ginsburg, Verlag Chemie: Weinheim, 1976.

[4] G. R. Desiraju, T. Steiner, 'The weak hydrogen bond in structural chemistry and biology', Oxford University Press Inc., New York, 1999.

[5] G. R. Desiraju, Nature 2001, 412, 397.

[6] (a) G. A. Jeffrey, Acta Cryst., Sect. B: Struct. Sci. 1990, 46, 89; (b) G. A. Jeffrey, W. Saenger, 'Hydrogen bonding in biological structures', Springer, Berlin, 1991; (c) C. André, P. Lurger, J.-H. Fuhrhop, B. Rosengarten, Acta Cryst., Sect. B: Struct. Sci. 1993, 49, 375; (d) G. A. Jeffrey, 'An Introduction to Hydrogen Bonding', Oxford University Press, Oxford, UK, 1997; (e) A. Bonnet, J. Chisholm, W. D. S. Motherwell, W. Jones, CrystEngComm. 2005, 7, 71.

[7] J. M. Robertson, 'Organic Crystals and Molecules', Cornell University Press, Ithaca, NY, 1953.

[8] R. Taylor, C. F. Macrae, Acta Cryst., Sect. B: Struct. Sci. 2001, 57, 815.

[9] C. P. Brock, Acta Cryst., Sect. B: Struct. Sci. 2002, 58, 1025.

[10] V. T. Nguyen, P. D. Ahn, R. Bishop, M. L. Scudder, D. C. Craig, Eur. J. Org. Chem. 2001, 4489.

[11] (a) E. S. Gardiner, A. Sarko, Can. J. Chem. 1985, 63, 173; (b) D. W. M. Hofmann, T. Lengauer, J. Mol. Model. 1998, 4, 132; (c) B. P. van Eijck, J. Kroon, J. Comput. Chem. 1999, 20, 799; (d) R. J. Viëtor, K. Mazeau, M. Lakin, S. Pérez, Biopolymers 2000, 54, 342; (e) B. P. van Eijck, W. T. M. Mooij, J. Kroon, J. Phys. Chem. B 2001, 105, 10573.
[12] G. Mehta, S. S. Ramesh, M. K. Bera, Chem. Eur. J. 2003, 9, 2264.

[13] (a) G. Mehta, D. S. Reddy, S. S. Ramesh, U. Tatu, Tetrahedron Lett. 1999, 40, 9141; (b) G. Mehta, S. S. Ramesh, Chem. Commun. 2000, 2429; (c) G. Mehta, S. S. Ramesh, Tetrahedron Lett. 2001, 42, 1987; (d) G. Mehta, S. S. Ramesh, Can. J. Chem. 2005, 83, 581.

[14] I. N. Rabinowitz, J. Kraut, Acta Crystallogr. 1964, 17, 159.

[15] G. Mehta, S. S. Ramesh, Eur. J. Org. Chem. 2005, 2225.

[16] G. Mehta, S. S. Ramesh, Tetrahedron Lett. 2003, 44, 3105

[17] G. Mehta, S. Sen, Tetrahedron Lett. 2010, 51, 503

[18] (a) G. Mehta, S. Sen, S. Dey, Acta Crystallogr., Sect. E: Struct. Rep. Online 2005, E61, o920; (b) G. Mehta, S. Sen, S. Dey, Acta Crystallogr., Sect. C: Cryst. Struct. Commun. 2005, C61, 0358 .

[19] G. A. Jeffrey, H. S. Kim, Acta Cryst., Sect. B: Struct. Sci. 1971, B27, 1812.

[20] G. Mehta, S. Sen, K. Venkatesan, CrystEngComm. 2005, 7, 656.

[21] G. Mehta, S. Sen, CrystEngComm. 2005, 7, 398.

[22] G. Mehta, S. Sen, S. S. Ramesh, Eur. J. Org. Chem. 2007, 423

[23] (a) W. Hückel, H. Schlee, Chem. Ber. 1955, 88, 346; (b) C. A. Grob, P. W. Schiess, Helv. Chim. Acta 1960, 43, 1546; (c) A. Shani, F. Sondheimer, J. Am. Chem. Soc. 1967, 89, 6310.

[24] G. Mehta, S. Sen, K. Venkatesan, CrystEngComm. 2007, 9, 144.

[25] (a) C. P. Brock, B. Schweizer, J. D. Dunitz, J. Am. Chem. Soc. 1991, 113, 9811; (b) M. C. Etter, K.-S. Huang, Chem. Mater. 1992, 4, 824; (c) A. Gavezzotti, Synlett 2002, 201; (d) M. S. Hendi, P. Hooter, R. E. Davis, V. M. Lynch, K. A. Wheeler, Cryst. Growth Des. 2004, 4, 95.

[26] C. P. Brock, J. D. Dunitz, Chem. Mater. 1994, 6 , 1118.

[27] J. Bernstein, 'Polymorphism in Molecular Crystals', Oxford University Press, Oxford, UK, 2002.

[28] G. Mehta, S. Sen, Chem. Commun. 2009, 5981.

[29] (a) E. Staab, L. Addadi, L. Leiserowitz, M. Lahav, Adv. Mater. 1990, 2, 40; (b) I. Weissbuch, L. Addadi, M. Lahav, L. Leiserowitz, Science 1991, 253, 637; (c) I. Weissbuch, L. Leiserowitz, M. Lahav, $A d v$. Mater. 1994, 6, 952; (d) I. Weissbuch, R. Popovitz-Biro, M. Lahav, L. Leiserowitz, Acta Cryst., Sect. B: Struct. Sci. 1995, 51, 115; (e) R. J. Davey, N. Blagden, G. D. Potts, R. Docherty, J. Am. Chem. Soc. 1997, 119, 1767; (f) I. Weissbuch, M. Lahav, L. Leiserowitz, in 'Advances in Crystal Growth Research', Eds. K. Sato, Y. Furukawa, K. Nakajima, Elsevier Science B.V., Amsterdam, 2001, pp. 381-400; (g) V. Yu. Torbeev, E. Shavit, I. Weissbuch, L. Leiserowitz, M. Lahav, Cryst. Growth Des. 2005, 5, 2190; h) W. J. P. van Enckevort, J. H. Los, J. Phys. Chem. C 2008, 112, 6380; (i) C. Murali, M. S. Shashidhar, R. G. Gonnade, M. M. Bhadbhade, Chem.-Eur. J. 2009, 15, 261.

[30] (a) A. Fürst, P. A. Plattner, Helv. Chim. Acta 1949, 32, 275; (b) A. Fürst, P. A. Plattner, Abstr. Papers Int. Congr. Pure Appl. Chem. 12 ${ }^{\text {th }}$, New York, 1951, 409.

[31] G. Mehta, S. Sen, Tetrahedron 2009, 65, 9713.
[32] G. Mehta, S. Sen, Acta Crystallogr., Sect. C: Cryst. Struct. Commun. 2010, C66, o59.

[33] (a) T. Hiyama, 'Organofluorine Compounds: Chemistry and Applications', SpringerVerlag, Berlin, 2000; (b) P. Kirsch, 'Modern Fluoroorganic Chemistry: Synthesis, Reactivity, Applications', WILEY-VCH, Weinheim, 2004; (c) H.-J. Böhm, D. Banner, S. Bendels, M. Kansy, B. Kuhn, K. Müller, U. Obst-Sander, M. Stahl, ChemBioChem 2004, 5, 637; (d) J. C. Biffinger, H. W. Kim, S. G. DiMagno, ChemBioChem 2004, 5, 622; (e) P. Maienfisch, R. G. Hall, Chimia 2004, 58, 93; (f) J.-P. Bégué, D. Bonnet-Delpon, J. Fluorine Chem. 2006, 127, 992; (g) K. Müller, C. Faeh, F. Diederich, Science 2007, 317, 1881.

[34] (a) J. A. K. Howard, V. J. Hoy, D. O'Hagan, G. T. Smith, Tetrahedron 1996, 52, 12613; (b) J. D. Dunitz, R. Taylor, Chem.-Eur. J. 1997, 3, 89; (c) J. D. Dunitz, ChemBioChem 2004, 5, 614; (d) K. Reichenbaecher, H. I. Suess, J. Hulliger, Chem. Soc. Rev. 2005, 34, 22; (e) J. D. Dunitz, W. B. Schweizer, Chem.-Eur. J. 2006, 12, 6804; (f) F. Cozzi, S. Bacchi, G. Filippini, T. Pilati, A. Gavezzotti, Chem.-Eur. J. 2007, 13, 7177; (g) D. Chopra, T. N. Guru Row, CrystEngComm 2011, 13, 2175; (h) H.-J. Schneider, Chem. Sci. 2012, 3, 1381.

[35] A. Bondi, J. Phys. Chem. 1964, 68, 441.

[36] G. Mehta, S. Sen, Eur. J. Org. Chem. 2010, 3387.

[37] (a) I. Shahak, S. Manor, E. D. Bergmann, J. Chem. Soc. (C) 1968, 2129; (b) R. Deghenghi, R. Gaudry, Can. J. Chem. 1961, 39, 1553; (c) A. I. Ayi, M. Remli, R. Condom, R. Guedj, J. Fluorine Chem. 1981, 17, 565; (d) A. Siwicka, D. Cuperly, L. Tedeschi, R. Le Vézouët, A. J. P. White, A. G. M. Barrett, Tetrahedron 2007, 63, 5903.

[38] The lack of stereoselectivity in the epoxidation of $\mathbf{3 5}$ contrasts the stereoselection that leads to the anti-diepoxide $\mathbf{2 0}$ as the sole product during the epoxidation of the diol 19. Formation of a $\mathrm{H}$-bonded complex between two molecules of mCPBA and the trans-hydroxy groups in $\mathbf{1 9}$ might account for the stereoselective formation of $\mathbf{2 0}$ (see ref. [22])

[39] Our attempts to isolate the monofluoropentol 31 from the reaction mixture via its peracetylation and subsequent transesterification of the purified tetraacetate derivative were repeatedly unsuccessful. Formation of a (trans, anti, trans, anti, trans)-perhydro-2,3,4a,6,7,8anaphthalenehexol during these endeavors was explained and proven on the basis of a base induced fluoride displacement in the initially formed 31, followed by a preferential anti-FürstPlattner opening by $\mathrm{OH}^{-}$in the epoxytetrol intermediate. For details, see: G. Mehta, S. Sen, J. Org. Chem. 2010, 75, 8287.

[40] (a) E. Vogel, M. Biskup, A. Vogel, H. Gunther, Angew. Chem., Int. Ed. Engl. 1966, 5, 734; (b) P. J. Garratt, F. Sondheimer, J. Chem. Soc. (C) 1967, 565

[41] G. Mehta, S. Sen, M. Thangam, Acta Crystallogr., Sect. C: Cryst. Struct. Commun. 2011, C67, o294.

[42] G. Mehta, S. Sen, Acta Crystallogr., Sect. E: Struct. Rep. Online 2011, E67, o1985.

[43] C. Jäckel, W. Seufert, S. Thust, B. Koksch, ChemBioChem 2004, 5, 717. 